\title{
Quantum communication through a spin chain with interaction determined by a Jacobi matrix
}

\author{
R Chakrabarti ${ }^{1}$ and $J$ Van der Jeugt \\ Department of Applied Mathematics and Computer Science, Ghent University, \\ Krijgslaan 281-S9, B-9000 Gent, Belgium \\ E-mail: ranabir@imsc.res.in and Joris.VanderJeugt@UGent.be
}

Received 4 December 2009, in final form 14 January 2010

Published 8 February 2010

Online at stacks.iop.org/JPhysA/43/085302

\begin{abstract}
We obtain the time-dependent correlation function describing the evolution of a single spin excitation state in a linear spin chain with isotropic nearestneighbour $X Y$ coupling, where the Hamiltonian is related to the Jacobi matrix of a set of orthogonal polynomials. For the Krawtchouk polynomial case, an arbitrary element of the correlation function is expressed in a simple closed form. Its asymptotic limit corresponds to the Jacobi matrix of the Charlier polynomial, and may be understood as a unitary evolution resulting from a Heisenberg group element. Correlation functions for Hamiltonians corresponding to Jacobi matrices for the Hahn, dual Hahn and Racah polynomials are also studied. For the Hahn polynomials we obtain the general correlation function, some of its special cases and the limit related to the Meixner polynomials, where the $\operatorname{su}(1,1)$ algebra describes the underlying symmetry. For the cases of dual Hahn and Racah polynomials, the general expressions of the correlation functions contain summations which are not of hypergeometric type. Simplifications, however, occur in special cases.
\end{abstract}

PACS numbers: $03.67 . \mathrm{Hk}, 02.30 . \mathrm{Gp}$

\section{Introduction}

Transfer of a known or an unknown quantum state from one site to another is a key requirement in linking neighbouring small quantum processors for facilitating large-scale quantum computation. Bose [1,2] introduced linear spin chains as a channel for such shortdistance quantum communication. Such a connector has an inherent advantage as it renders the quantum processor and the communicating channel to be made of the same physical

1 Permanent address: Department of Theoretical Physics, University of Madras, Guindy Campus, Chennai 600025 , India. 
system. This eliminates the need of developing interfaces. Transmission of data in such linear quantum registers has been the subject of many investigations [1, 3-6]. Using the classical concept of group velocity of a wavepacket, it has been observed [7] that one-dimensional spin rings allow high-fidelity transmission of quantum states if both communicating parties have access to a finitely limited number of qubits in the ring. Quantum communication with closed spin ring with twisted boundary conditions has been discussed in [2]. An excellent review [8] and references therein describe the current developments in this field.

The transmission of quantum states can in principle be performed by a chain of qubits coupled via the Heisenberg or the $X Y$ interactions [9-12]. Interesting situations arise if one assumes to have individual control of the nearest-neighbour couplings in the spin chain. The idea of pre-engineered interqubit couplings has been discussed considerably [13, 14]. One of the advantages of well-chosen controlled couplings is that one can obtain mirror inversion of a quantum state with respect to the centre of the chain, and that perfect transfer of quantum states is possible $[3,6,15]$ at certain specified times over arbitrary length of the spin chain. Propagation of entangled states in anisotropic $X Y$ spin chains has been discussed in [16]. Using a system based on a dispersive qubit-boson interaction to mimic $X Y$ coupling that relaxes the nearest-neighbour restriction, the transfer fidelity of the chain has been found [5] to achieve a nearly optimal value.

One of the main results of [15] is the introduction of two (analytic) mirror-periodic Hamiltonians that allow for perfect transfer. To achieve perfect mirror inversion of an arbitrary many excitation state, it is sufficient to consider transformations of all single excitation states to their mirror images as the time-dependent transition amplitudes for multiple excitation states may be constructed $[15,17]$ via the Slater determinant of their single excitation counterparts. The eigenstates of the single excitation sectors of these Hamiltonians are related [15] to discrete orthogonal polynomials, namely Krawtchouk polynomials and dual Hahn polynomials.

In the present paper we investigate such systems from a general point of view. We will assume that the chain of qubits is described by a Hamiltonian of $X Y$ type, in such a way that the interaction strengths (and qubit energies) are related to the Jacobi matrix of a system of discrete orthogonal polynomials. This will allow us to give a general expression for the transition amplitude of a single excitation from the sth site (sending site) to the $r$ th site (receiving site) in the chain of spins. For some types of discrete orthogonal polynomials, the expression of these transition amplitudes can be simplified, leading to some interesting properties.

Let us consider a, by now, classical system of $N+1$ interacting qubits (spin $1 / 2$ particles) in a quantum register, with an isotropic Hamiltonian of $X Y$ type:

$$
\hat{H}=\frac{1}{2} \sum_{k=0}^{N-1} J_{k}\left(\sigma_{k}^{x} \cdot \sigma_{k+1}^{x}+\sigma_{k+1}^{y} \cdot \sigma_{k}^{y}\right)+\frac{1}{2} \sum_{k=0}^{N} h_{k}\left(\sigma_{k}^{z}+1\right),
$$

where $J_{k}$ is the coupling strength between the qubits located at sites $k$ and $k+1$, and $h_{k}$ is the 'Zeeman' energy of a qubit at site $k$. So the subindex $k(k=0,1,2, \ldots, N)$ labels the position of the qubit in the chain, and the superindex refers to the Pauli matrices $\sigma^{x}, \sigma^{y}$ and $\sigma^{z}$. Hamiltonian (1) preserves the total spin: $\left[\hat{H}, \sum_{k=0}^{N} \sigma_{k}^{z}\right]=0$, and, therefore, we may analyse various spin excitation sectors separately.

To describe the Hilbert space associated with the Hamiltonian, one adopts a standard fermionization technique [17]. The Jordan-Wigner transformation [18] maps the Pauli matrices to spinless lattice fermions $\left\{a_{k}, a_{k}^{\dagger} \mid k=0,1, \ldots, N\right\}$ obeying the anticommutation rules

$\left\{a_{k}^{\dagger}, a_{\ell}\right\}=\delta_{k, \ell}, \quad\left\{a_{k}, a_{\ell}\right\}=\left\{a_{k}^{\dagger}, a_{\ell}^{\dagger}\right\}=0 \quad \forall k, \ell \in\{0,1, \ldots, N\}$. 
We may now recast Hamiltonian (1) as

$$
\hat{H}=\sum_{k=0}^{N-1} J_{k}\left(a_{k}^{\dagger} a_{k+1}+a_{k+1}^{\dagger} a_{k}\right)+\sum_{k=0}^{N} h_{k} a_{k}^{\dagger} a_{k}
$$

that describes a set of $N+1$ fermions on a chain with nearest-neighbour interaction (hopping between adjacent sites of the chain), and subject to a non-uniform background magnetic field denoted by $h_{k}(k=0,1, \ldots, N)$. We will assume that the system is initially in its completely polarized ground state $|\mathbf{0}\rangle=|00 \cdots 0\rangle=|0\rangle \otimes|0\rangle \otimes \cdots \otimes|0\rangle$, where $|0\rangle$ denotes the spin down state. Let $\mid k)=|00 \cdots 010 \cdots 0\rangle=a_{k}^{\dagger}|\mathbf{0}\rangle(k=0,1, \ldots, N)$ denote a state in which there is a single fermion at the site $k$ and all other sites are empty, i.e. $\mid k)$ describes the state in which the spin at the site $k$ has been flipped to $|1\rangle$. Clearly, the set of states $\mid k)(k=0,1, \ldots, N)$ forms a basis for the single-fermion states of the system, and we may represent them by the standard unit vectors in the column matrix form

$$
k)=\left(\begin{array}{c}
0 \\
0 \\
\vdots \\
1 \\
\vdots \\
0
\end{array}\right) \quad(k=0,1, \ldots, N)
$$

In this single-fermion basis, the Hamiltonian $\hat{H}$ takes the matrix form

$$
M=\left(\begin{array}{ccccc}
h_{0} & J_{0} & 0 & \ldots & 0 \\
J_{0} & h_{1} & J_{1} & \ldots & 0 \\
0 & J_{1} & h_{2} & \ddots & \\
\vdots & \vdots & \ddots & \ddots & J_{N-1} \\
0 & 0 & & J_{N-1} & h_{N}
\end{array}\right)
$$

The dynamics (time evolution) of the system is completely determined by the eigenvalues $\epsilon_{j}$ and eigenvectors $\phi_{j}$ of this matrix. It is then, as noted before, a standard technique [15, 17] to describe the $n$-fermion eigenstates of $\hat{H}(n \leqslant N)$ using the single-fermion eigenstates $\phi_{j}$ and Slater determinants. For this reason, we concentrate here on the single-fermion eigenstates.

The matrix $M$ in (5) is real and symmetric, so the spectral theorem [19] implies that it can be written as

$$
M=U D U^{T}
$$

where $D$ is a diagonal matrix and $U$ an orthogonal matrix:

$$
\begin{aligned}
& D=\operatorname{diag}\left(\epsilon_{0}, \epsilon_{1}, \ldots, \epsilon_{N}\right) \\
& U U^{T}=U^{T} U=I
\end{aligned}
$$

The entries of $D$ are the single-fermion energy eigenvalues, and the columns of the matrix $U$ are the (orthonormal) eigenvectors of $M$, i.e. the single-fermion eigenstates

$$
\left.\phi_{j}=\left(\begin{array}{c}
U_{0 j} \\
U_{1 j} \\
\vdots \\
U_{N j}
\end{array}\right)=\sum_{k=0}^{N} U_{k j} \mid k\right)=\sum_{k=0}^{N} U_{k j} a_{k}^{\dagger}|\mathbf{0}\rangle \quad(j=0,1, \ldots, N)
$$


with $\hat{H} \phi_{j}=M \phi_{j}=\epsilon_{j} \phi_{j}$. From the orthogonality of $U$, the inverse relation follows:

$$
\mid k)=\sum_{j=0}^{N} U_{k j} \phi_{j}
$$

We now turn to the dynamics of the system under consideration, described by the unitary time evolution operator

$$
\mathcal{U}(t) \equiv \exp (-\mathrm{i} t \hat{H})
$$

Assume that the 'state sender' is located at site $s$ of the spin chain, and the 'state receiver' at site $r$ ( $s$ and $r$ are site labels, belonging to $\{0,1, \ldots, N\}$ ). At time $t=0$, the sender turns the system into the single spin state $\mid s$ ). After a certain time $t$, the system evolves to the state $\mathcal{U}(t) \mid s)$ that may be expressed as a linear superposition of all the single spin states. So the transition amplitude of an excitation from site $s$ to site $r$ of the spin chain is given by the time-dependent correlation function

$$
f_{r, s}(t)=(r|\mathcal{U}(t)| s)
$$

This is the central quantity of this paper (sometimes referred to as the 'correlation function'), and the square of its modulus gives the transition probability from the $s$ th to the $r$ th spin excitation state. Note that it can be expressed by means of the orthogonal matrix $U$ appearing in (6). Indeed, using definition (12), expansion (10), and the orthogonality of the states $\phi_{j}$, one finds

$$
\begin{aligned}
f_{r, s}(t) & =\left\langle\sum_{k=0}^{N} U_{r k} \phi_{k} \mid \exp (-\mathrm{i} t \hat{H}) \sum_{j=0}^{N} U_{s j} \phi_{j}\right\rangle \\
& =\left\langle\sum_{k=0}^{N} U_{r k} \phi_{k} \mid \sum_{j=0}^{N} U_{s j} \mathrm{e}^{-\mathrm{i} t \epsilon_{j}} \phi_{j}\right\rangle \\
& =\sum_{j=0}^{N} U_{r j} U_{s j} \mathrm{e}^{-\mathrm{i} t \epsilon_{j}} .
\end{aligned}
$$

In other words, using the abbreviation $z=\mathrm{e}^{-\mathrm{i} t}$, one has

$$
f_{r, s}(t)=\sum_{j=0}^{N} U_{r j} U_{s j} z^{\epsilon_{j}} \quad\left(z=\mathrm{e}^{-\mathrm{i} t}\right) .
$$

The purpose of this paper is to show that various interesting closed form expressions can be given for this crucial quantity $f_{r, s}(t)$, in the case when the fixed values characterizing the system (the values $J_{k}$ and $h_{k}$ ) are related to the Jacobi matrix of a set of discrete orthogonal polynomials. We will illustrate this first by means of an example, where the polynomials are Krawtchouk polynomials. From this example, the general technique will be clear. Then we continue analysing some systems related to other classes of orthogonal polynomials.

From the outset it is assumed that such chains of qubits can be pre-engineered for any given set of values $J_{k}$ and $h_{k}$, i.e. the strength of the couplings can be engineered and the external static potential at site $k$ is controlled. Such a physical control has been the subject of many papers (see [3] and references therein).

We end this section by two remarks. First of all, the strengths $J_{k}$ in (3), and thus the offdiagonal elements of $M$ in (5), are positive. From the mathematical point of view, however, the 
problem can just as well be solved for a matrix with negative off-diagonal elements. Indeed, if

$$
M^{\prime}=\left(\begin{array}{ccccc}
h_{0} & -J_{0} & 0 & \ldots & 0 \\
-J_{0} & h_{1} & -J_{1} & \ldots & 0 \\
0 & -J_{1} & h_{2} & \ddots & \\
\vdots & \vdots & \ddots & \ddots & -J_{N-1} \\
0 & 0 & & -J_{N-1} & h_{N}
\end{array}\right)
$$

then $M_{j k}^{\prime}=(-1)^{j+k} M_{j k}$. This implies that $M^{\prime}$ has the same eigenvalues $\epsilon_{j}$ as $M$. Moreover, the orthogonal matrix $U^{\prime}$ with $U_{j k}^{\prime}=(-1)^{j+k} U_{j k}$ diagonalizes $M^{\prime}$ in the same way as in (6), so the eigenvectors of $M^{\prime}$ (i.e. the columns of $U^{\prime}$ ) are the same as those of $M$ up to sign changes in the components. Then the transition amplitude follows from (14) and is essentially the same as that corresponding to $M: f_{r, s}^{\prime}(t)=(-1)^{r+s} f_{r, s}(t)$. Secondly, also a matrix that differs from the original one by a constant factor and a multiple of the identity matrix leads essentially to the same computation. Indeed, let $M^{\prime}=\lambda M+\mu I$, where $\lambda$ and $\mu$ are constants and $I$ is the identity matrix. Then the same matrix $U$ from (6) diagonalizes $M^{\prime}$, the only difference being the eigenvalues given now by $\lambda \epsilon_{j}+\mu$. Also, it follows immediately from (14) that $f_{r, s}^{\prime}(t)=\mathrm{e}^{-\mathrm{i} t \mu} f_{r, s}(\lambda t)$.

\section{The Jacobi matrix of Krawtchouk polynomials}

\subsection{Computation of the general correlation function}

Let us start this section by introducing some standard notation and known facts of Krawtchouk polynomials. We follow the notation of [20]; other standard works on (discrete) orthogonal polynomials are $[21,22]$. The Krawtchouk polynomial of degree $n(n=0,1, \ldots, N)$ in the variable $x$, with parameter $0<p<1$ is given by

$$
K_{n}(x) \equiv K_{n}(x ; p, N)={ }_{2} F_{1}\left(\begin{array}{c}
-x,-n \\
-N
\end{array} ; \frac{1}{p}\right) .
$$

The function ${ }_{2} F_{1}$ is the classical hypergeometric series [23, 24], and in this case it is a terminating series because of the appearance of the negative integer $-n$ as a numerator parameter. Krawtchouk polynomials satisfy a (discrete) orthogonality relation [20]:

$$
\sum_{x=0}^{N} w(x) K_{n}(x) K_{m}(x)=d_{n} \delta_{m n}
$$

where $w(x)$ is the weight function in $x$ and $d_{n}$ is a function depending on $n$ :

$$
w(x)=\left(\begin{array}{l}
N \\
x
\end{array}\right) p^{x}(1-p)^{N-x} \quad(x=0,1, \ldots, N) ; \quad d_{n}=\frac{1}{\left(\begin{array}{l}
N \\
n
\end{array}\right)}\left(\frac{1-p}{p}\right)^{n} .
$$

They also satisfy the following three-term recurrence relation:

$$
-x K_{n}(x)=n(1-p) K_{n-1}(x)-[p(N-n)+n(1-p)] K_{n}(x)+p(N-n) K_{n+1}(x) .
$$

It is often convenient to introduce orthonormal Krawtchouk functions by

$$
\tilde{K}_{n}(x) \equiv \frac{\sqrt{w(x)} K_{n}(x)}{\sqrt{d_{n}}} .
$$

Then, rewriting the orthogonality relation and the recurrence relation in terms of the functions $\tilde{K}_{n}(x)$, it is easy to obtain the following. 
Lemma 1 (see [25]). Let $M_{K}$ be the tridiagonal $(N+1) \times(N+1)$-matrix (Jacobi matrix)

$$
M_{K}=\left(\begin{array}{ccccc}
h_{0} & -J_{0} & 0 & & \\
-J_{0} & h_{1} & -J_{1} & \ddots & \\
0 & -J_{1} & h_{2} & \ddots & 0 \\
& \ddots & \ddots & \ddots & -J_{N-1} \\
& & 0 & -J_{N-1} & h_{N}
\end{array}\right)
$$

where

$$
J_{n}=\sqrt{p(1-p)} \sqrt{(n+1)(N-n)}, \quad h_{n}=N p+(1-2 p) n,
$$

and let $U$ be the $(N+1) \times(N+1)$-matrix with elements $U_{j k}=\tilde{K}_{j}(k)$. Then

$$
U U^{T}=U^{T} U=I \quad \text { and } \quad M_{K}=U D U^{T},
$$

where

$$
D=\operatorname{diag}(0,1,2, \ldots, N)
$$

In other words, the eigenvectors of the Hamiltonian (in the single-fermion case) corresponding to the quantities (22) have components equal to normalized Krawtchouk polynomials, and the corresponding energy eigenvalues are $\epsilon_{j}=j(j=0,1, \ldots, N)$. Note that Krawtchouk polynomials have been used before as a basis for quantum chains [26]. Here, only their evaluations at integer values of the support are used as matrix elements of $U$ in the diagonalization process.

Let us now consider the transition amplitude or correlation function:

$$
\begin{aligned}
f_{r, s}(t) & =\sum_{k=0}^{N} U_{r k} U_{s k} z^{\epsilon_{k}}=\sum_{k=0}^{N} \tilde{K}_{r}(k) \tilde{K}_{s}(k) z^{k} \\
& =\frac{1}{\sqrt{d_{r} d_{s}}} \sum_{k=0}^{N} w(k) K_{r}(k) K_{s}(k) z^{k} \quad\left(z=\mathrm{e}^{-\mathrm{i} t}\right) .
\end{aligned}
$$

So we need to compute the quantity in (25). First of all, note that in general $f_{r, s}(t)$ is a periodic function of $t$ since $z=\mathrm{e}^{-\mathrm{i} t}$. In particular, it follows from (25) and the orthogonality relation (17) that $f_{r, s}(t)=\delta_{r s}$ for $t=0$ and for any multiple of $2 \pi$. So after a time span of $2 \pi$, the system is back to its original state where only the spin at the sending site $s$ is flipped.

The purpose is now to compute (25) explicitly. We will do this in two ways: a classical method and a group theoretical method. The classical method is short and straightforward. Rewriting the polynomials in (25) as ${ }_{2} F_{1}$-series, this sum reduces to a classical summation formula given for example in $[27, \mathrm{p} 84,(8)]$. This leads immediately to the following closed form expression:

$$
\begin{gathered}
f_{r, s}(t)=\sqrt{\left(\begin{array}{c}
N \\
r
\end{array}\right)\left(\begin{array}{c}
N \\
s
\end{array}\right)}(\sqrt{p(1-p)})^{r+s}(1-z)^{r+s}(1-p+p z)^{N-r-s} \\
\quad \times{ }_{2} F_{1}\left(\begin{array}{c}
-r,-s \\
-N
\end{array} ; \frac{-z}{p(1-p)(1-z)^{2}}\right) .
\end{gathered}
$$

Before discussing some special and interesting cases of this formula, we will also deduce this correlation function in a different way. 


\subsection{Group-theoretical computation}

The group-theoretical way to obtain (26) is somewhat longer, but it does not use any reference to orthogonal polynomials or summation formulas of hypergeometric type. So it sheds another light on why the final formula (26) is so simple.

Consider the Lie algebra $s u(2)$ of quantum angular momentum theory [28], with basis $L_{0}, L_{ \pm}$and commutation relations

$$
\left[L_{0}, L_{ \pm}\right]= \pm L_{ \pm}, \quad\left[L_{+}, L_{-}\right]=2 L_{0} .
$$

For $N$ any positive integer, the $(N+1)$-dimensional irreducible representation is given by

$$
\begin{aligned}
L_{0} & =\left(\begin{array}{ccccc}
\frac{N}{2} & & & & \\
& \frac{N}{2}-1 & & & \\
& & \ddots & & \\
& & & -\frac{N}{2}+1 & \\
& & & & -\frac{N}{2}
\end{array}\right), \\
L_{+} & =\left(\begin{array}{ccccc}
0 & \sqrt{1 \cdot N} & & \\
& 0 & \sqrt{2 \cdot(N-1)} & \\
& & \ddots & & \\
& & & & 0
\end{array}\right),
\end{aligned}
$$

and $L_{-}=\left(L_{+}\right)^{T}$. Note that, in this representation, the matrix $M_{K}$ from (21) is written as

$$
M_{K}=\frac{N}{2} I+(2 p-1) L_{0}-\sqrt{p(1-p)}\left(L_{+}+L_{-}\right),
$$

where $I$ is the identity matrix. Consequently, the computation of

$$
f_{r, s}(t)=\left(r\left|\exp \left(-\mathrm{i} t M_{K}\right)\right| s\right)
$$

simply leads to the computation of a matrix element of an $S U(2)$ group element. So apart from a factor $\mathrm{e}^{-\mathrm{i} t N / 2}$, we need the computation of the following left-hand side, which we write as

$$
\exp \left(-\mathrm{i} t(2 p-1) L_{0}+\mathrm{i} t \sqrt{p(1-p)}\left(L_{+}+L_{-}\right)\right)=\mathrm{e}^{\xi L_{-}} \mathrm{e}^{\eta L_{0}} \mathrm{e}^{\zeta L_{+}} .
$$

Equation (31) is an example of the standard Baker-Campbell-Hausdorff (BCH) type of decomposition of an $S U$ (2) group element. For a general discussion on $\mathrm{BCH}$ decomposition of group elements see [29]. As we intend to use such decompositions in other contexts, we provide here the usual procedure of derivation of these rules. The constants $\xi, \eta, \zeta$ in (31) should be representation independent (all elements are group elements), so to determine these we can perform the computation using any faithful representation, and, in particular, the standard two-dimensional representation. Expanding the exponential in the left-hand side of (31), using the $2 \times 2$ matrices

$$
L_{0} \rightarrow\left(\begin{array}{cc}
1 / 2 & 0 \\
0 & -1 / 2
\end{array}\right), \quad L_{+} \rightarrow\left(\begin{array}{ll}
0 & 1 \\
0 & 0
\end{array}\right), \quad L_{-} \rightarrow\left(\begin{array}{ll}
0 & 0 \\
1 & 0
\end{array}\right),
$$

one obtains after some calculations

$$
\left(\begin{array}{cc}
\cos \frac{t}{2}-\mathrm{i}(2 p-1) \sin \left(\frac{t}{2}\right) & 2 \mathrm{i} \sqrt{p(1-p)} \sin \left(\frac{t}{2}\right) \\
2 \mathrm{i} \sqrt{p(1-p)} \sin \left(\frac{t}{2}\right) & \cos \left(\frac{t}{2}\right)+\mathrm{i}(2 p-1) \sin \left(\frac{t}{2}\right)
\end{array}\right) .
$$


On the other hand, the right-hand side of (31) yields, in the same two-dimensional representation (32)

$$
\left(\begin{array}{cc}
\mathrm{e}^{\eta / 2} & \zeta \mathrm{e}^{\eta / 2} \\
\xi \mathrm{e}^{\eta / 2} & \xi \zeta \mathrm{e}^{\eta / 2}+\mathrm{e}^{-\eta / 2}
\end{array}\right)
$$

Identification yields

$\mathrm{e}^{\eta / 2}=\cos \left(\frac{t}{2}\right)-\mathrm{i}(2 p-1) \sin \left(\frac{t}{2}\right), \quad \xi=\zeta=\frac{2 \mathrm{i} \sqrt{p(1-p)} \sin \left(\frac{t}{2}\right)}{\cos \left(\frac{t}{2}\right)-\mathrm{i}(2 p-1) \sin \left(\frac{t}{2}\right)}$.

Now we compute the matrix element of the right-hand side of (31) for an arbitrary representation of dimension $(N+1)$. Using the common matrix elements $(28)$, one finds

$\left.\left.\left.\left.L_{0} \mid s\right)=\left(\frac{N}{2}-s\right) \mid s\right), \quad \frac{\left(L_{+}\right)^{k}}{k !} \mid s\right)=\sqrt{\left(\begin{array}{l}s \\ k\end{array}\right)\left(\begin{array}{c}N-s+k \\ k\end{array}\right)} \mid s-k\right)$,

and similarly

$$
\left(r \mid \frac{\left(L_{-}\right)^{j}}{j !}=\sqrt{\left(\begin{array}{l}
r \\
j
\end{array}\right)\left(\begin{array}{c}
N-r+j \\
j
\end{array}\right)}(r-j \mid .\right.
$$

Now (using $\xi=\zeta$ )

$$
\begin{aligned}
\left(r\left|\mathrm{e}^{\xi L_{-}} \mathrm{e}^{\eta L_{0}} \mathrm{e}^{\zeta L_{+}}\right| s\right) & =\sum_{j, k=0}^{N} \frac{\xi^{j+k}}{j ! k !}\left(r\left|\left(L_{-}\right)^{j} \mathrm{e}^{\eta L_{0}}\left(L_{+}\right)^{k}\right| s\right) \\
& =\sum_{j, k} \xi^{j+k} \sqrt{\left(\begin{array}{l}
r \\
j
\end{array}\right)\left(\begin{array}{c}
N-r+j \\
j
\end{array}\right)\left(\begin{array}{l}
s \\
k
\end{array}\right)\left(\begin{array}{c}
N-s+k \\
k
\end{array}\right)} \mathrm{e}^{\eta\left(\frac{N}{2}-s+k\right)} \delta_{r-j, s-k} \\
& =\sum_{j} \sqrt{\left(\begin{array}{l}
r \\
j
\end{array}\right)\left(\begin{array}{c}
N-r+j \\
j
\end{array}\right)\left(\begin{array}{c}
s \\
s-r+j
\end{array}\right)\left(\begin{array}{c}
N-r+j \\
s-r+j
\end{array}\right)} \xi^{s-r+2 j} \mathrm{e}^{\eta\left(\frac{N}{2}-r+j\right)} \\
& =\sqrt{\frac{r ! s !}{(N-r) !(N-s) !}} \xi^{s-r} \mathrm{e}^{\eta\left(\frac{N}{2}-r\right)} \sum_{j} \frac{(N-r+j) !}{j !(r-j) !(j+s-r) !}\left(\xi^{2} \mathrm{e}^{\eta}\right)^{j}
\end{aligned}
$$

The last sum is of hypergeometric type and is proportional to

$$
{ }_{2} F_{1}\left(\begin{array}{c}
-r,-s \\
-N
\end{array} ; \frac{-1}{\xi^{2} e^{\eta}}\right)={ }_{2} F_{1}\left(\begin{array}{c}
-r,-s \\
-N
\end{array} ; \frac{1}{4 p(1-p) \sin ^{2}\left(\frac{t}{2}\right)}\right),
$$

using (35). But $z=\mathrm{e}^{-\mathrm{i} t}$, so $(1-z)^{2} / z=-4 \sin ^{2}\left(\frac{t}{2}\right)$, and taking all factors together one recovers (26). Note that this computation is essentially equivalent to that relating matrix elements of finite rotations ( $d$-functions) to Jacobi polynomials [28, chapter 4$]$.

\subsection{Discussion and special cases}

Let us return to the closed form expression (26) for the transition amplitude $f_{r, s}(t)$ at time $t$. As already noted, since $f_{r, s}(t)$ is a function of $z=\mathrm{e}^{-\mathrm{i} t}$, the system is periodic with period $2 \pi$. At the initial moment $t=0$, the system is in the state with all spins down except at site $s$ where the spin is up. At any time which is a multiple of $2 \pi$, the system is back to this initial 
state: $f_{r, s}(2 k \pi)=\delta_{r s}$ for $k \in \mathbb{Z}_{+}$. At other times, the system is in general in a mixed state. Due to the orthogonality of the basis states, one has

$$
\sum_{r=0}^{N}\left|f_{r, s}(t)\right|^{2}=1
$$

for any $s$ and any time $t$. In fact, more generally, the matrix of correlation functions is unitary, so for any time $t$ and any indices $r$ and $s$

$$
\sum_{k=0}^{N} f_{k, r}^{*}(t) f_{k, s}(t)=\sum_{k=0}^{N} f_{r, k}^{*}(t) f_{s, k}=\delta_{r, s} .
$$

Let us consider the case when the sender is located at site 0 , i.e. $s=0$. Then (26) yields

$$
f_{r, 0}(t)=\sqrt{\left(\begin{array}{c}
N \\
r
\end{array}\right)}(\sqrt{p(1-p)})^{r}(1-z)^{r}(1-p+p z)^{N-r} .
$$

So far, $p(0<p<1)$ is still a free parameter. A special case occurs when $p=1 / 2$ :

$$
f_{r, 0}(t)=\frac{1}{2^{N}} \sqrt{\left(\begin{array}{c}
N \\
r
\end{array}\right)}(1-z)^{r}(1+z)^{N-r}, \quad(p=1 / 2) .
$$

Using $z=\mathrm{e}^{-\mathrm{i} t}$, this gives

$$
\left|f_{r, 0}(t)\right|=\sqrt{\left(\begin{array}{c}
N \\
r
\end{array}\right)}\left|\sin \left(\frac{t}{2}\right)\right|^{r}\left|\cos \left(\frac{t}{2}\right)\right|^{N-r}, \quad(p=1 / 2) .
$$

In other words,

$$
f_{r, 0}(\pi)=\delta_{r, N}, \quad(p=1 / 2) .
$$

This is the situation of 'perfect state transfer' described already in [15]: at time $t=\pi$ the system is in the state with all spins down except at site $N$ where the spin is up. So for this time there is perfect state transfer from site 0 to site $N$.

Let us mention here that the condition for perfect state transfer can be deduced from the corresponding Jacobi matrix itself [6]. In order to allow perfect state transfer, matrix (5) should be mirror-periodic, i.e. $h_{n}=h_{N-n}$ and $J_{n}=J_{N-1-n}$ for all $n$. Clearly, for (21) this is the case when $p=1 / 2$, see (22).

More generally, let us specialize expression (26) for time $t=\pi$ :

$f_{r, s}(\pi)=\sqrt{\left(\begin{array}{c}N \\ r\end{array}\right)\left(\begin{array}{c}N \\ s\end{array}\right)}(\sqrt{p(1-p)})^{r+s} 2^{r+s}(1-2 p)^{N-r-s}{ }_{2} F_{1}\left(\begin{array}{c}-r,-s \\ -N\end{array} ; \frac{1}{4 p(1-p)}\right)$.

This expression shows once again that taking the free parameter $p=1 / 2$ yields a special case:

$$
f_{r, s}(\pi)=\delta_{r+s, N}, \quad(p=1 / 2) .
$$

So for $p=1 / 2$ there is also perfect state transfer between the sites $s$ and $N-s$.

\subsection{A limiting case}

A classical limit of Krawtchouk polynomials is Charlier polynomials. Putting the parameter $p=\alpha / N$, and letting $N$ go to $+\infty$, yields Charlier polynomials $C_{n}(x ; \alpha)$ [20]:

$$
\lim _{N \rightarrow+\infty} K_{n}\left(x ; \frac{\alpha}{N}, N\right)=C_{n}(x ; \alpha)={ }_{2} F_{0}\left(\begin{array}{c}
-n,-x \\
-
\end{array} ;-\frac{1}{\alpha}\right),
$$


satisfying the orthogonality relations

$$
\sum_{x=0}^{\infty} \frac{\alpha^{x}}{x !} \mathrm{e}^{-\alpha} C_{m}(x ; \alpha) C_{n}(x ; \alpha)=\frac{n !}{\alpha^{n}} \delta_{m n},
$$

where $\alpha>0$ is a positive parameter. The recurrence relation reads

$$
x C_{n}(x ; \alpha)=-\alpha C_{n+1}(x ; \alpha)+(n+\alpha) C_{n}(x ; \alpha)-n C_{n-1}(x ; \alpha) .
$$

To see which spin chain corresponds to this limit, we take the appropriate limit in (22), and find for (3):

$$
\hat{H}=\sum_{k=0}^{\infty}(\alpha+k) a_{k}^{\dagger} a_{k}-\sum_{k=0}^{\infty} \sqrt{\alpha(k+1)}\left(a_{k}^{\dagger} a_{k+1}+a_{k+1}^{\dagger} a_{k}\right) .
$$

The above infinite chain of fermions with nearest-neighbour interaction immediately provides a unitary representation of the (central extension of the) Heisenberg algebra $\mathfrak{h}_{4}\left(\mathfrak{a}, \mathfrak{a}^{\dagger}, \mathfrak{N}, \mathfrak{I}\right)$, where the algebraic generators may be constructed as

$\mathfrak{a}=\sum_{k=0}^{\infty} \sqrt{k+1} a_{k}^{\dagger} a_{k+1}, \quad \mathfrak{a}^{\dagger}=\sum_{k=0}^{\infty} \sqrt{k+1} a_{k+1}^{\dagger} a_{k}, \quad \mathfrak{N}=\sum_{k=0}^{\infty} k a_{k}^{\dagger} a_{k}, \quad \mathfrak{I}=\sum_{k=0}^{\infty} a_{k}^{\dagger} a_{k}$.

Employing the defining anticommutation relations of the fermionic variables (2), we observe that the above operators satisfy the commutation relations of the Heisenberg algebra $\mathfrak{h}_{4}\left(\mathfrak{a}, \mathfrak{a}^{\dagger}, \mathfrak{N}, \mathfrak{I}\right)$ :
$\left[\mathfrak{a}, \mathfrak{a}^{\dagger}\right]=\mathfrak{I}$,
$[\mathfrak{N}, \mathfrak{a}]=-\mathfrak{a}$,
$\left[\mathfrak{N}, \mathfrak{a}^{\dagger}\right]=\mathfrak{a}^{\dagger}$
$[\mathfrak{X}, \mathfrak{I}]=0 \quad$ where $\mathfrak{X} \in\left\{\mathfrak{N}, \mathfrak{a}, \mathfrak{a}^{\dagger}\right\}$,

where the unitary representation in terms of the single spin excitation states (10) reads

$\left.\left.\left.\left.\left.\mathfrak{a}^{\dagger} \mid k\right)=\sqrt{k+1} \mid k+1\right), \quad \mathfrak{a} \mid k\right)=\sqrt{k}(k-1), \quad \mathfrak{N} \mid k\right)=k \mid k\right)$.

Using the generators introduced in (49), we may express Hamiltonian (48) as

$$
\hat{H}=\mathfrak{N}+\alpha \mathfrak{I}-\sqrt{\alpha}\left(\mathfrak{a}+\mathfrak{a}^{\dagger}\right) .
$$

Following [29], the time evolution operator introduced in (11) may now be expressed in the normal ordered $\mathrm{BCH}$-factorized form

$\mathcal{U}(t)=\exp (\alpha(z-1)) \exp \left(\sqrt{\alpha}(1-z) \mathfrak{a}^{\dagger}\right) z^{\mathfrak{N}} \exp (\sqrt{\alpha}(1-z) \mathfrak{a})$

It is worth mentioning that the correlation function (12) obtained via the operator factorization (53) and the appropriate limiting value easily computed from (26) precisely agree. We quote the final answer:

$$
f_{r, s}(t)=\sqrt{\frac{\alpha^{r+s}}{r ! s !}}(1-z)^{r+s} \mathrm{e}^{-\alpha+\alpha z}{ }_{2} F_{0}\left(\begin{array}{c}
-r,-s \\
-
\end{array} \frac{z}{\alpha(1-z)^{2}}\right)
$$

Intuitively the above result is easily understood from the well-known [29] result that the large spin $\left(\frac{N}{2}\right)$ contraction limit of the $s u(2)$ algebra (27) is given by the Heisenberg algebra. From (54), it follows that the state with spin up at position $s=0$ emanates over the infinite chain with the correlation function given below

$$
f_{r, 0}(t)=\sqrt{\frac{\alpha^{r}}{r !}}\left(1-\mathrm{e}^{-\mathrm{i} t}\right)^{r} \mathrm{e}^{-\alpha+\alpha \exp (-\mathrm{i} t)} .
$$


Starting at time $t=0$, some reflection takes place at the 'infinite end' of the chain, and by time $t=2 \pi$ the system is back to its original state. In the middle of this, at time $t=\pi$, the state is 'spread' over the infinite chain according to the amplitude

$$
f_{r, 0}(\pi)=\mathrm{e}^{-2 \alpha} \sqrt{\frac{(4 \alpha)^{r}}{r !}} .
$$

Note that $\sum_{r=0}^{\infty} f_{r, 0}^{2}(\pi)=1$, as it should be. The asymptotic limiting value of the correlation function obtained above may be understood in the sense of the leading term in a $\frac{1}{N}$ expansion of the said quantity (26) for the fixed $N$ case. It should be interesting to obtain successive correction terms, and their group theoretic interpretations, to the leading value in the large $N$ limit.

\section{The Jacobi matrix of Hahn polynomials}

\subsection{General correlation function and special cases}

The method outlined in the beginning of the previous section is clear, and this analysis can in principle be made for any set of discrete orthogonal polynomials. So in this section we will consider the more general class of Hahn polynomials $Q_{n}(x ; \alpha, \beta, N)$ [20, 21], characterized by a positive integer parameter $N$ and two real parameters $\alpha$ and $\beta$ (for orthogonality, one should have $\alpha>-1$ and $\beta>-1$, or $\alpha<-N$ and $\beta<-N$ ). The Hahn polynomial of degree $n(n=0,1, \ldots, N)$ in the variable $x$ is defined by

$Q_{n}(x) \equiv Q_{n}(x ; \alpha, \beta, N)={ }_{3} F_{2}\left(\begin{array}{c}-n, n+\alpha+\beta+1,-x \\ \alpha+1,-N\end{array}\right)$.

The orthogonality relation reads

$$
\sum_{x=0}^{N} w(x) Q_{n}(x) Q_{m}(x)=d_{n} \delta_{m n}
$$

where

$$
\begin{aligned}
& w(x)=\left(\begin{array}{c}
\alpha+x \\
x
\end{array}\right)\left(\begin{array}{c}
N+\beta-x \\
N-x
\end{array}\right) \quad(x=0,1, \ldots, N) ; \\
& d_{n}=\frac{n !(N-n) !}{N !^{2}} \frac{(n+\alpha+\beta+1)_{N+1}(\beta+1)_{n}}{(2 n+\alpha+\beta+1)(\alpha+1)_{n}} .
\end{aligned}
$$

We have used the common notation for hypergeometric series and Pochhammer symbols $[23,24]$, like $(a)_{n}=a(a+1) \cdots(a+n-1)$ for $n=1,2, \ldots$ and $(a)_{0}=1 ;(a, b, \ldots)_{n}=$ $(a)_{n}(b)_{n} \cdots$, etc. The three-term recurrence relation is given by

$$
-x Q_{n}(x)=A_{n} Q_{n+1}(x)-\left(A_{n}+C_{n}\right) Q_{n}(x)+C_{n} Q_{n-1}(x),
$$

where

$$
A_{n}=\frac{(n+\alpha+\beta+1)(n+\alpha+1)(N-n)}{(2 n+\alpha+\beta+1)(2 n+\alpha+\beta+2)}, \quad C_{n}=\frac{n(n+\alpha+\beta+N+1)(n+\beta)}{(2 n+\alpha+\beta)(2 n+\alpha+\beta+1)} .
$$

Introducing orthonormal Hahn functions

$$
\tilde{Q}_{n}(x) \equiv \frac{\sqrt{w(x)} Q_{n}(x)}{\sqrt{d_{n}}},
$$

one has the following result [25]. 
Lemma 2. Let $M_{Q}$ be the tridiagonal $(N+1) \times(N+1)$-matrix (Jacobi matrix)

$$
M_{Q}=\left(\begin{array}{ccccc}
h_{0} & -J_{0} & 0 & & \\
-J_{0} & h_{1} & -J_{1} & \ddots & \\
0 & -J_{1} & h_{2} & \ddots & 0 \\
& \ddots & \ddots & \ddots & -J_{N-1} \\
& & 0 & -J_{N-1} & h_{N}
\end{array}\right)
$$

where

$J_{n}=\sqrt{\frac{(n+1)(n+\alpha+1)(n+\beta+1)(n+\alpha+\beta+1)(n+\alpha+\beta+N+2)(N-n)}{(2 n+\alpha+\beta+2)^{2}(2 n+\alpha+\beta+1)(2 n+\alpha+\beta+3)}}$,

$h_{n}=\frac{N}{2}+\frac{(\alpha-\beta)[(\alpha+\beta)(N-2 n)-2 n(n+1)]}{2(2 n+\alpha+\beta)(2 n+\alpha+\beta+2)}$.

and let $U$ be the $(N+1) \times(N+1)$-matrix with elements $U_{j k}=\tilde{Q}_{j}(k)$. Then

$$
U U^{T}=U^{T} U=I \quad \text { and } \quad M_{Q}=U D U^{T},
$$

where

$$
D=\operatorname{diag}(0,1,2, \ldots, N)
$$

So for a system corresponding to the quantities (61), the transition amplitude is given by

$$
f_{r, s}(t)=\frac{1}{\sqrt{d_{r} d_{s}}} \sum_{k=0}^{N} w(k) Q_{r}(k) Q_{s}(k) z^{k} \quad\left(z=\mathrm{e}^{-\mathrm{i} t}\right) .
$$

The purpose is now to compute (64), and then to investigate some special cases. Let us denote the summation in (64) by $S(r, s)$ :

$$
S(r, s)=\sum_{k=0}^{N} w(k) Q_{r}(k) Q_{s}(k) z^{k}
$$

In order to perform this summation, one can use the following product formula for Hahn polynomials:

$$
\begin{gathered}
Q_{r}(k) Q_{s}(k)={ }_{3} F_{2}\left(\begin{array}{c}
-k,-r, r+\alpha+\beta+1 \\
-N, \alpha+1
\end{array} ; 1\right){ }_{3} F_{2}\left(\begin{array}{c}
-k,-s, s+\alpha+\beta+1 \\
-N, \alpha+1
\end{array} ; 1\right) \\
=\frac{(-N-\beta)_{k}}{(\alpha+1)_{k}} \sum_{m=0}^{k} \frac{(-k, r-N, s-N,-r-c,-s-c)_{m}}{(1,-N-\beta,-c,-N,-N)_{m}} \\
\times{ }_{8} F_{7}\left(\begin{array}{c}
c-m, 1+\frac{c-m}{2}, N+\beta+1-m,-m,-r,-s, c+r-N, c+s-N \\
\frac{c-m}{2}, \alpha+1, c+1, c+1+r-m, c+1+s-m, N+1-r-m, N+1-s-m
\end{array} ;-1\right),
\end{gathered}
$$

where we have used the abbreviation $c=N+1+\alpha+\beta$. This expression can be obtained from the product formula for $q$-Racah polynomials given in [30, equation (8.3.1)]: in this formula, first take the limit $a \rightarrow 0$, and then take the limit $q \rightarrow 1$.

Now we multiply the right-hand side of (66) by $w(k) z^{k}$, and sum over $k$ from 0 to $N$. Changing the order of summation (over $k$ and $m$ ), the inner sum over $k$ can be performed using 
the binomial theorem. This leads to

$$
\begin{aligned}
S(r, s)=\frac{(\beta+1)_{N}}{N !} \sum_{m=0}^{N}(-z)^{m}(1-z)^{N-m}{ }_{8} F_{7}(-1) \\
\quad \times \frac{(r-N, s-N,-r-N-\alpha-\beta-1,-s-N-\alpha-\beta-1)_{m}}{m !(-N,-N-\beta,-N-\alpha-\beta-1) m},
\end{aligned}
$$

where the ${ }_{8} F_{7}(-1)$ has the same parameters as in (66). So together with the factor $1 / \sqrt{d_{r} d_{s}}$ in (64), (67) gives us a symmetric and compact formula for the computation of $f_{r, s}(t)$ in the Hahn case.

Let us consider the special case when the sender is at one end of the chain, i.e. $s=0$. Then (67) gives

$$
\begin{aligned}
S(r, 0) & =\frac{(\beta+1)_{N}}{N !} \sum_{m}(-z)^{m}(1-z)^{N-m} \frac{(r-N,-r-N-\alpha-\beta-1)_{m}}{m !(-N-\beta) m} \\
& =\frac{(\beta+1)_{N}}{N !}(1-z)^{N}{ }_{2} F_{1}\left(\begin{array}{c}
r-N,-r-N-\alpha-\beta-1 \\
-N-\beta
\end{array} \frac{z}{z-1}\right) \\
& =\frac{(\beta+1)_{N}}{N !}(1-z)^{r}{ }_{2} F_{1}\left(\begin{array}{c}
r-N, r+\alpha+1 \\
-N-\beta
\end{array} ;\right) .
\end{aligned}
$$

In the last step, Euler's transformation [23, 24] formula was used. Thus, the transition amplitude becomes

$$
\begin{aligned}
f_{r, 0}(t)=\left(\left(\begin{array}{c}
N \\
r
\end{array}\right) \frac{(2 r+\alpha+\beta+1)(\alpha+1)_{r}}{(\beta+1)_{r}(\alpha+\beta+2)_{N}(r+\alpha+\beta+1)_{N+1}}\right)^{1 / 2} \\
\quad \times(\beta+1)_{N}(1-z)^{r}{ }_{2} F_{1}\left(\begin{array}{c}
r-N, r+\alpha+1 \\
-N-\beta
\end{array} ; z\right) .
\end{aligned}
$$

In particular,

$$
f_{N, 0}(t)=\left(\frac{(\alpha+1, \beta+1)_{N}}{(\alpha+\beta+2)_{N}(N+\alpha+\beta+1)_{N}}\right)^{1 / 2}(1-z)^{N}
$$

and

$$
\left|f_{N, 0}(t)\right|=\left(\frac{(\alpha+1, \beta+1)_{N}}{(\alpha+\beta+2)_{N}(N+\alpha+\beta+1)_{N}}\right)^{1 / 2} 2^{N}\left|\sin \left(\frac{t}{2}\right)\right|^{N} .
$$

An interesting special case is that with the parameters $\alpha$ and $\beta$ equal, because then the magnetic field strengths $h_{k}$ in (3) are all constant (independent of $k$ ), see (61). For $\beta=\alpha$, (71) becomes

$$
\left|f_{N, 0}(t)\right|=\left(\frac{(\alpha+1)_{N}}{(\alpha+3 / 2)_{N-1}(\alpha+N / 2+1 / 2)}\right)^{1 / 2}\left|\sin \left(\frac{t}{2}\right)\right|^{N}
$$

Clearly, this is maximal for $t=\pi$ (plus multiples of $2 \pi$ ). However, $\left|f_{N, 0}(t)\right|<1$ for the allowed values of $\alpha$. Only for large $\alpha,\left|f_{N, 0}(t)\right|$ approaches 1 . So 'perfect state transfer' between site 0 and site $N$ does not take place except for $\alpha \rightarrow+\infty$. This limiting case does not give rise to a new example: for $\alpha=\beta \rightarrow+\infty$, the Hahn polynomials reduce to Krawtchouk polynomials with $p=1 / 2$, and this was the subject of the previous section.

\subsection{A limiting case}

A classical limit of Hahn polynomials is Meixner polynomials. Putting $\alpha=b-1, \beta=N \frac{1-c}{c}$ and letting $N$ go to $+\infty$ yields Meixner polynomials $M_{n}(x ; b, c)$ [20]:

$$
\lim _{N \rightarrow+\infty} Q_{n}\left(x ; b-1, N \frac{1-c}{c}, N\right)=M_{n}(x ; b, c)={ }_{2} F_{1}\left(\begin{array}{c}
-n,-x \\
b
\end{array} 1-\frac{1}{c}\right),
$$


satisfying the orthogonality relations

$$
\sum_{x=0}^{\infty} \frac{(b)_{x}}{x !} c^{x} M_{m}(x ; b, c) M_{n}(x ; b, c)=\frac{c^{-n} n !}{(b)_{n}(1-c)^{b}} \delta_{m n}
$$

where $b>0$ is a positive parameter and $0<c<1$. For the recurrence relation, see [20].

Again one can wonder which spin chain corresponds to this limit. Taking the appropriate limits in (61), one finds for (3):

$$
\hat{H}=\sum_{k=0}^{\infty} \frac{k+c(k+b)}{1-c} a_{k}^{\dagger} a_{k}-\sum_{k=0}^{\infty} \frac{\sqrt{c(k+1)(k+b)}}{1-c}\left(a_{k}^{\dagger} a_{k+1}+a_{k+1}^{\dagger} a_{k}\right) .
$$

It may be immediately observed that the $s u(1,1)$ algebra acts as the spectrum generating algebra of the above Hamiltonian. We define the $\operatorname{su}(1,1)$ generators and the identity operator as bilinear constructs of the fermionic variables by

$$
\begin{aligned}
& \mathcal{K}_{0}=\sum_{k=0}^{\infty}\left(k+\frac{b}{2}\right) a_{k}^{\dagger} a_{k}, \\
& \mathcal{K}_{+}=\sum_{k=0}^{\infty} \sqrt{(k+1)(k+b)} a_{k+1}^{\dagger} a_{k}, \\
& \mathcal{K}_{-}=\sum_{k=0}^{\infty} \sqrt{(k+1)(k+b)} a_{k}^{\dagger} a_{k+1}, \\
& \mathcal{I}=\sum_{k=0}^{\infty} a_{k}^{\dagger} a_{k} .
\end{aligned}
$$

By virtue of (2), the above generators satisfy the $s u(1,1)$ commutation relations:

$$
\left[\mathcal{K}_{0}, \mathcal{K}_{ \pm}\right]= \pm \mathcal{K}_{ \pm}, \quad\left[\mathcal{K}_{+}, \mathcal{K}_{-}\right]=-2 \mathcal{K}_{0}, \quad[\mathcal{X}, \mathcal{I}]=0 \quad \text { where } \quad \mathcal{X} \in\left\{\mathcal{K}_{0}, \mathcal{K}_{ \pm}\right\}
$$

The large $N$ Hamiltonian (75) now assumes the form

$$
\hat{H}=\frac{1+c}{1-c} \mathcal{K}_{0}-\frac{b}{2} \mathcal{I}-\frac{\sqrt{c}}{1-c}\left(\mathcal{K}_{+}+\mathcal{K}_{-}\right) .
$$

The infinite-dimensional lowest-weight representation of $s u(1,1)$ reads (see, e.g. [31, equation (2.2)] or [32])

$$
\begin{aligned}
& \left.\left.\mathcal{K}_{+} \mid \frac{b}{2}, n\right)=\sqrt{(n+1)(n+b)} \mid \frac{b}{2}, n+1\right), \\
& \left.\left.\mathcal{K}_{-} \mid \frac{b}{2}, n\right)=\sqrt{n(n+b-1)} \mid \frac{b}{2}, n-1\right), \\
& \left.\left.\mathcal{K}_{0} \mid \frac{b}{2}, n\right)=\left(n+\frac{b}{2}\right) \mid \frac{b}{2}, n\right),
\end{aligned}
$$

where the lowest weight $\frac{b}{2}$ has been made explicit in the notation of the state vector. Following [29], the BCH-factorization of the time evolution operator (11) may now be easily obtained:

$\mathcal{U}(t)=\frac{1}{\sqrt{z^{b}}} \exp \left(\sqrt{c} \frac{1-z}{1-c z} \mathcal{K}_{+}\right)\left(\frac{(1-c) \sqrt{z}}{1-c z}\right)^{2 \mathcal{K}_{0}} \exp \left(\sqrt{c} \frac{1-z}{1-c z} \mathcal{K}_{-}\right)$. 
Employing the decomposition (80), the correlation function (12) for the asymptotic limit of the spin chain governed by Hamiltonian (78) is readily obtained. As expected, this precisely agrees with the appropriate limit that can easily be computed from (67):

$f_{r, s}(t)=(1-c)^{b} \sqrt{\frac{(b)_{r}(b)_{s}}{r ! s !}} c^{(r+s) / 2} \frac{(1-z)^{r+s}}{(1-c z)^{b+r+s}}{ }^{2} F_{1}\left(\begin{array}{c}-r,-s \\ b\end{array} ; c\left(1-\frac{1}{c}\right)^{2} \frac{z}{(1-z)^{2}}\right)$.

As noted in section 2.4, the above asymptotic limit of the transition amplitude (64) may be understood as its leading term in a $\frac{1}{N}$ expansion scheme. Let us consider an example here, say for $b=1$ and $c=1 / 2$. At time $t=0$, the state with spin up at position $s=0$ is 'released' over the infinite chain; then at time $t=\pi$ it is 'spread' as follows:

$$
f_{r, 0}(\pi)=\frac{1}{3}\left(\frac{\sqrt{8}}{3}\right)^{r}
$$

(verify that $\sum_{r=0}^{\infty} f_{r, 0}^{2}(\pi)=1$ ). So it decays exponentially over the chain, only to return back to its original configuration at time $t=2 \pi$.

\section{The Jacobi matrix of dual Hahn and Racah polynomials}

\subsection{General computation for dual Hahn polynomials}

Dual Hahn polynomials will play a special role. First of all, the energy spectrum is not linear. Secondly, under certain conditions they will allow perfect state transfer.

Dual Hahn polynomials $R_{n}(\lambda(x) ; \gamma, \delta, N)[20,21]$ are characterized by a positive integer parameter $N$ and two real parameters $\gamma$ and $\delta$ (for orthogonality, one should have $\gamma>-1$ and $\delta>-1$, or $\gamma<-N$ and $\delta<-N)$. The dual Hahn polynomial is not a polynomial of degree $n$ in $x$, but of degree $n(n=0,1, \ldots, N)$ in $\lambda(x)=x(x+\gamma+\delta+1)$ :

$$
R_{n}(\lambda(x)) \equiv R_{n}(\lambda(x) ; \gamma, \delta, N)={ }_{3} F_{2}\left(\begin{array}{c}
-n,-x, x+\gamma+\delta+1 \\
\gamma+1,-N
\end{array} ; 1\right)
$$

The orthogonality relation reads

$$
\sum_{x=0}^{N} w(x) R_{n}(\lambda(x)) R_{m}(\lambda(x))=d_{n} \delta_{m n},
$$

where

$$
\begin{aligned}
& w(x)=\frac{(2 x+\gamma+\delta+1)(\gamma+1)_{x}(-N)_{x} N !}{(-1)^{x}(x+\gamma+\delta+1)_{N+1}(\delta+1)_{x} x !} \quad(x=0,1, \ldots, N) \\
& d_{n}^{-1}=\left(\begin{array}{c}
\gamma+n \\
n
\end{array}\right)\left(\begin{array}{c}
\delta+N-n \\
N-n
\end{array}\right) .
\end{aligned}
$$

The three-term recurrence relation is given by

$\lambda(x) R_{n}(\lambda(x))=A_{n} R_{n+1}(\lambda(x))-\left(A_{n}+C_{n}\right) R_{n}(\lambda(x))+C_{n} R_{n-1}(\lambda(x))$,

where

$$
A_{n}=(n+\gamma+1)(n-N), \quad C_{n}=n(n-\delta-N-1) .
$$

Orthonormal dual Hahn functions are defined by

$$
\tilde{R}_{n}(\lambda(x)) \equiv \frac{\sqrt{w(x)} R_{n}(\lambda(x))}{\sqrt{d_{n}}},
$$


and then one can deduce:

Lemma 3. Let $M_{R}$ be the tridiagonal $(N+1) \times(N+1)$-matrix (Jacobi matrix)

$$
M_{R}=\left(\begin{array}{ccccc}
h_{0} & -J_{0} & 0 & & \\
-J_{0} & h_{1} & -J_{1} & \ddots & \\
0 & -J_{1} & h_{2} & \ddots & 0 \\
& \ddots & \ddots & \ddots & -J_{N-1} \\
& & 0 & -J_{N-1} & h_{N}
\end{array}\right),
$$

where

$$
\begin{aligned}
& J_{n}=\sqrt{(n+1)(n+\gamma+1)(N-n)(\delta+N-n)}, \\
& h_{n}=(n+\gamma+1)(N-n)+n(\delta+N-n+1) .
\end{aligned}
$$

and let $U$ be the $(N+1) \times(N+1)$-matrix with elements $U_{j k}=\tilde{R}_{j}(\lambda(k))$. Then

$$
U U^{T}=U^{T} U=I \quad \text { and } \quad M_{R}=U D U^{T}
$$

where

$$
D=\operatorname{diag}\left(\epsilon_{0}, \epsilon_{1}, \epsilon_{2}, \ldots, \epsilon_{N}\right) \quad \text { with } \quad \epsilon_{k}=k(k+\gamma+\delta+1)
$$

Note that due to the appearance of $\lambda(x)$ in (85), the matrix $D$ has the form (90), and thus the energy eigenvalues of the single-fermion Hamiltonian eigenstates are quadratic in $k$.

The rest of the analysis is again concerned with the general correlation function. For a system corresponding to the quantities (88), this is now given by

$$
f_{r, s}(t)=\frac{1}{\sqrt{d_{r} d_{s}}} \sum_{k=0}^{N} w(k) R_{r}(\lambda(k)) R_{s}(\lambda(k)) z^{k(k+\gamma+\delta+1)} \quad\left(z=\mathrm{e}^{-\mathrm{i} t}\right) .
$$

In this case, one can use the product formula for $q$-Hahn polynomials [30, equation (8.3.3)] and take the limit $q \rightarrow 1$ to find

$$
\begin{aligned}
R_{r}(\lambda(k)) R_{S}(\lambda(k)) & ={ }_{3} F_{2}\left(\begin{array}{c}
-r,-k, k+\gamma+\delta+1 \\
\gamma+1,-N
\end{array} ; 1\right){ }_{3} F_{2}\left(\begin{array}{c}
-s,-k, k+\gamma+\delta+1 \\
\gamma+1,-N
\end{array} ;\right) \\
& =(-1)^{k} \frac{(\delta+1)_{k}}{(\gamma+1)_{k}} \sum_{m=0}^{k} \frac{(-k, r-N, s-N, \gamma+\delta+k+1)_{m}}{(1, \delta+1,-N,-N)_{m}} \\
& \times{ }_{4} F_{3}\left(\begin{array}{c}
-m,-r,-s,-\delta-m \\
\gamma+1, N+1-r-m, N+1-s-m
\end{array} ; 1\right) .
\end{aligned}
$$

Then one obtains, using (92) and exchanging the order of summation,

$$
\begin{aligned}
f_{r, s}(t)=\frac{1}{\sqrt{d_{r} d_{s}}} \sum_{m=0}^{N} \frac{(r-N, s-N)_{m}}{(\delta+1)_{m}} F_{3}\left(\begin{array}{c}
-m,-r,-s,-\delta-m \\
\gamma+1, N+1-r-m, N+1-s-m
\end{array} ; 1\right) \\
\quad \times(-1)^{m} \frac{((N-m) !)^{2}}{m ! N !} \sum_{k=m}^{N} \frac{(-N)_{k}(\gamma+\delta+k+1)_{m}(\gamma+\delta+2 k+1)}{(k-m) !(\gamma+\delta+k+1)_{N+1}} z^{k(k+\gamma+\delta+1)} .
\end{aligned}
$$


Due to the appearance of $z^{k(k+\gamma+\delta+1)}$, the inner sum is no longer of hypergeometric type, and it cannot be simplified in general. Let us therefore specialize to the case with $s=0$ and $r=N$ (sending site at one end and receiving site at the other end of the chain). Then

$$
f_{N, 0}(t)=\sqrt{(\gamma+1, \delta+1)_{N}} \sum_{k=0}^{N} \frac{(-N)_{k}(\gamma+\delta+2 k+1)}{k !(\gamma+\delta+k+1)_{N+1}} z^{k(k+\gamma+\delta+1)} .
$$

So far, $\gamma$ and $\delta$ are free parameters. Let us now require the following condition: $\gamma+\delta$ is an odd integer number. Then at time $t=\pi$, one has $z^{k(k+\gamma+\delta+1)}=(-1)^{k(k+\gamma+\delta+1)}=(-1)^{k}$. The summation over $k$ in (94) can now be performed, since it corresponds to a nearly poised ${ }_{3} F_{2}(-1)$ (see [24, (III.25)]). One obtains

$$
f_{N, 0}(\pi)=\frac{\sqrt{(\gamma+1, \delta+1)_{N}}}{\left(\frac{\gamma+\delta}{2}+1\right)_{N}}, \quad(\gamma+\delta=\text { odd integer }) .
$$

Clearly, this expression assumes its maximum value for $\gamma=\delta$, and in that case it is equal to 1. In other words, for $\gamma=\delta=p+\frac{1}{2}$, with $p$ an integer, there is perfect state transfer between the sites 0 and $N$ at time $t=\pi$.

Similarly, when $\gamma+\delta$ is of the form

$$
\gamma+\delta=\frac{2 p+1}{q}, \quad p, q \in \mathbb{N} \quad(q \neq 0),
$$

it also follows that at time $t=q \pi$,

$$
z^{k(k+\gamma+\delta+1)}=\mathrm{e}^{-\mathrm{i} q \pi\left[k(k+1)+\frac{2 p+1}{q} k\right]}=\mathrm{e}^{-\mathrm{i} \pi(2 p+1) k}=(-1)^{k} .
$$

So in that case, $f_{N, 0}(q \pi)$ assumes the same value as given by the right-hand side of (95). Thus, for $\gamma=\delta=\frac{2 p+1}{2 q}$, one has perfect state transfer from 0 to $N$ at time $t=q \pi$. This is the situation described in [15].

\subsection{The case of Racah polynomials}

Dual Hahn polynomials are a limiting case of Racah polynomials. Among the discrete orthogonal polynomials, Racah polynomials are the most general, but also the most complicated. Their Jacobi matrix is not mirror-periodic, so perfect state transfer is not possible [6]. Racah polynomials $R_{n}(\lambda(x) ; \alpha, \beta, \gamma, \delta)$ are polynomials of degree $n$ in the variable $\lambda(x)=x(x+\gamma+\delta+1)$, and are expressed as a ${ }_{4} F_{3}(1)$ series, where one of the numerator parameters $\alpha+1, \beta+\delta+1$ or $\gamma+1$ should be $-N$, with $N$ a positive integer [20]. Without loss of generality, let us assume that we are in the first case with $\alpha+1=-N$. Then, for the weight function to be positive, assume

$$
\gamma+1>0, \quad \delta+1>0, \quad \beta>\gamma+N
$$

The orthogonality relation and the coefficients of the recurrence relation become fairly complicated, see [20]. Moreover, the single-fermion eigenvalues are of the same form as (90). This means that the correlation function is given by (91), where $w(k)$ and $d_{n}$ now stand for the weight function and squared norm of the Racah polynomials respectively, and where $R_{n}(\lambda(k))$ is a Racah polynomial. Due to the appearance of $z^{k(k+\gamma+\delta+1)}$, the final summation is again no longer of hypergeometric type and cannot be simplified in general. Let us therefore not give the general expression, but only some special cases. For $s=0$ and $r=N$, the correlation function becomes

$f_{N, 0}(t)=\frac{1}{\sqrt{d_{N} d_{0}}} \sum_{k=0}^{N} \frac{(-N, \gamma+\delta+1,(\gamma+\delta+1) / 2+1)_{k}}{k !(\gamma+\delta+N+2,(\gamma+\delta+1) / 2)_{k}} z^{k(k+\gamma+\delta+1)}$. 
When $z^{k(k+\gamma+\delta+1)}=(-1)^{k}$, this sum becomes a nearly poised ${ }_{3} F_{2}(-1)$ series which can be summed using [24, (III.25)]. In other words, when $\gamma+\delta$ is an odd integer number, one finds

$$
f_{N, 0}(\pi)=\sqrt{\frac{(\gamma+1-\beta, \delta+1+\beta)_{N}}{(\beta,-\beta)_{N}}} \frac{\sqrt{(\gamma+1, \delta+1)_{N}}}{\left(\frac{\gamma+\delta}{2}+1\right)_{N}} .
$$

Note that in the limit $\beta \rightarrow+\infty$, in which case the Racah polynomials become dual Hahn polynomials, (99) indeed becomes (95).

\section{Discussion and conclusion}

In this paper, we have considered linear spin chains with a nearest-neighbour hopping interaction, as models for quantum communication. We have considered the time evolution of single-fermion states in such a spin chain. In particular, if the system is at time $t=0$ in a pure state with all spins down except one spin up at site $s$ of the chain, we have studied the behaviour of this system at time $t$ by computing the transition amplitude $f_{r, s}(t)$. The main contribution of the paper is to show that one can deduce closed form expressions for this transition amplitude (or correlation function) if the interaction matrix of the system is related to the Jacobi matrix of a set of (discrete) orthogonal polynomials.

We have worked out in detail the cases related to Krawtchouk polynomials (section 2), Hahn polynomials (section 3) and dual Hahn polynomials (section 4); for the case of Racah polynomials we give only some partial result in section 4.2. Experts in orthogonal polynomials might wonder why we did not proceed the opposite way, starting from the most general case (Racah polynomials), and then obtaining the other cases as certain limits. This approach would work here only for the limit from Racah polynomials to dual Hahn polynomials, because of the appearance of $z^{k(k+\gamma+\delta+1)}$ in (98) and (91). For the other correlation functions, there is just $z^{k}$ in the summation part, and these need to be treated separately anyway. The Krawtchouk case could have been presented as a limit of the Hahn case; however, we felt that it was better to start with a simple example first, which moreover has some additional interesting properties (such as the group-theoretical interpretation, the special case of perfect state transfer and an interesting limit of its own). The case of Hahn and dual Hahn polynomials had to be considered separately because of the different nature of the correlation function $\left(z^{k}\right.$ as opposed to $\left.z^{k(k+\gamma+\delta+1)}\right)$.

For all examples considered here, we have obtained complete or partial results. In the case of Krawtchouk polynomials, we obtained a simple closed form expression for $f_{r, s}(t)$ in general. This was also the case for a limit consisting of an infinite chain of spins described by the Jacobi matrix of Charlier polynomials. We noted that this example is related to the unitary representation of the Heisenberg algebra. In the case of Hahn polynomials, we do obtain a general expression (67) for $f_{r, s}(t)$, though it is still quite complicated. Some special cases have been discussed, as well as the limit related to Meixner polynomials (where a simple general expression is obtained). We have related this example to the $s u(1,1)$ symmetry algebra of the corresponding Hamiltonian. The asymptotic $N \rightarrow \infty$ limit of the spin chain may be understood in the framework of a $\frac{1}{N}$ expansion where the leading terms of the correlation functions of the related Hamiltonians are obtained. In the case of dual Hahn polynomials, the general expression for $f_{r, s}(t)$ contains a summation part which is not of hypergeometric type, but we do show that it simplifies in special cases. The same remarks hold for the case of Racah polynomials.

It is worth discussing certain related areas where our analysis may find extensions or applications. Propagation of entangled states in anisotropic spin chains has been studied in 
[16]. Anisotropic models are characterized by the property that they allow instantaneous creation of pairwise entanglement from the fully polarized ground state. The anisotropy parameter connects [16] the isotropic $X Y$ model with the quantum Ising model, which, in the $N \rightarrow \infty$ limit, undergoes a quantum phase transition at a critical value of the coupling constant. In our case it should be of interest to understand the dynamics of propagation of entangled states in a spin chain governed by an anisotropic variation of Hamiltonian (1), where the coupling constants follow the polynomial structures considered here. A class of Hamiltonians that do not preserve the total number of excited spins was found [33] to dynamically create multipartite entangled states starting from an initially uncorrelated state. In this context, one may also introduce the one-axis spin squeezing interactions in the Hamiltonian [34] that is expected to protect the entangled states against decoherence. The group theoretic method developed in our work may help in describing analytical solutions for evolutions of such entangled states in the quantum register. Work towards this is in progress.

We complete our work mentioning another context in which the formalism developed here may be relevant. A recent work [35] considers a Jaynes-Cummings-Hubbard (JCH) system that describes coupled cavity structures where confined photons are induced to interact via their coupling to embedded two-state systems. In particular, a non-uniform 'parabolic' coupling between the cavities is assumed in [35] that is identical to our discussions in section 2 regarding the nearest-neighbour coupling guided by the Krawtchouk polynomials. The large $N$ asymptotic limit of the correlation function obtained in (54) may be useful in understanding the mean field results, and consequently, the quantum phase transitions for the JCH Hamiltonians. Moreover, our examples of Jacobi matrices corresponding to Hahn polynomials and their asymptotic limits, and the dual Hahn polynomials may also be considered as pre-engineered couplings between the cavities for JCH systems. Our evaluation of the correlation functions for these cases may have some importance in developing the theory of JCH systems with inter-cavity couplings subject to these polynomial structures.

\section{Acknowledgments}

This research was supported by project P6/02 of the Interuniversity Attraction Poles Programme (Belgian State-Belgian Science Policy). R Chakrabarti wishes to acknowledge Ghent University for a visitors grant. J Van der Jeugt wishes to thank M Rahman for pointing out the relevance of [30, equation (8.3.1)] in this context. We thank EI Jafarov for discussions and for pointing out [35] to us.

\section{References}

[1] Bose S 2003 Phys. Rev. Lett. 91207901

[2] Bose S, Jin B-Q and Korepin V E 2005 Phys. Rev. A 72022345

[3] Christandl M, Datta N, Ekert A and Landahl A J 2004 Phys. Rev. Lett. 92187902

[4] Christandl M, Datta N, Dorlas T C, Ekert A, Kay A and Landahl A J 2005 Phys. Rev. A 71032312

[5] Paternostro M, Palma G M, Kim M S and Falci G 2005 Phys. Rev. A 71042311

[6] Kay A 2009 A review of perfect state transfer and its application as a constructive tool arXiv:0903.4274v2

[7] Osborne T J and Linden N 2004 Phys. Rev. A 69052315

[8] Bose S 2007 Contemp. Phys. 48 13-30

[9] DiVincenzo D P, Bacon D, Kempe J, Burkard G and Whaley K B 2000 Nature 408 339-42

[10] Benjamin S C 2002 Phys. Rev. Lett. 88107904

[11] Zhou X X, Zhou Z W, Guo G C and Feldman M J 2002 Phys. Rev. Lett. 89197903

[12] Benjamin S C and Bose S 2003 Phys. Rev. Lett. 90247901

[13] Yung M H and Bose S 2005 Phys. Rev. A 71032310

[14] Karbach P and Stolze J 2005 Phys. Rev. A 72030301 
[15] Albanese C, Christandl M, Datta N and Ekert A 2004 Phys. Rev. Lett. 93230502

[16] Amico L, Osterloh A, Plastina F, Fazio R and Palma G M 2004 Phys. Rev. A 69022304

[17] Lieb E and Wu F 1968 Phys. Rev. Lett. $201445-8$

[18] Jordan P and Wigner E 1928 Z. Phys. 47631

[19] Golub G H and Van Loan C F 1996 Matrix Computations (Baltimore: Johns Hopkins University Press)

[20] Koekoek R and Swarttouw R F 1998 The Askey-scheme of hypergeometric orthogonal polynomials and its q-analogue (Technical Report 98-17, Delft University of Technology)

[21] Nikiforov A F, Suslov S K and Uvarov V B 1991 Classical Orthogonal Polynomials of a Discrete Variable (Berlin: Springer)

[22] Ismail M E H 2005 Classical and Quantum Orthogonal Polynomials in One Variable (Cambridge: Cambridge University Press)

[23] Bailey W N 1964 Generalized Hypergeometric Series (Cambridge: Cambridge University Press)

[24] Slater L J 1966 Generalized Hypergeometric Functions (Cambridge: Cambridge University Press)

[25] Regniers G and Van der Jeugt J 2009 J. Phys. A: Math. Theor. 42125301

[26] Atakishiev N A, Jafarov E I, Nagiyev S M and Wolf K B 1998 Rev. Mex. Fis. 44 235-44

[27] Erdélyi A, Magnus W, Oberhettinger F and Tricomi F G 1953 Higher Transcendental Functions vol 1 (New York: McGraw-Hill)

[28] Edmonds A R 1960 Angular Momentum in Quantum Theory (Princeton, NJ: Princeton University Press)

[29] Zhang W M, Feng D H and Gilmore R 1990 Rev. Mod. Phys. 62 867-927

[30] Gasper G and Rahman M 2004 Basic Hypergeometric Series (Cambridge: Cambridge University Press)

[31] Van der Jeugt J 1997 J. Math. Phys. 38 2727-40

[32] Bacry H 1990 J. Math. Phys. 31 2061-77

[33] Franco C D, Paternostro M, Tsomokos D I and Huelga S F 2008 Phys. Rev. A 77062337

[34] Kitagawa M and Ueda M 1993 Phys. Rev. A 47 5138-43

[35] Makin M I, Cole J H, Hill C D, Greentree A D and Hollenberg L C L 2009 Phys. Rev. A 80043842 